\title{
Atividade de Forrageamento de Formigas (Hymenoptera: Formicidae) em Áreas de Mata e Campo de Gramíneas no Pantanal sul-mato-grossense
}

\author{
Michelle Viscardi Sant’Ana ${ }^{1}$, Rose Benedita Rodrigues Trindade ${ }^{1}$, Cássia Cristina dos Santos Lopes ${ }^{1}$, \\ Odival Faccenda ${ }^{2} \&$ Wedson Desidério Fernandes ${ }^{1}$
}

1. Universidade Federal da Grande Dourados, e-mail: mivsbio@yahoo.com.br, rosetrinda@gmail.com (Autor para correspondência), crisinha_lopes@yahoo.com.br, wdf.entomo@ig.com.br, 2. Universidade Estadual de Mato Grosso do Sul, e-mail: fac@uems.br

EntomoBrasilis 1(2): 29-32 (2008)

Resumo. O principal objetivo do estudo foi avaliar a taxa de exploração de iscas vivas por formigas em um Ambiente de Mata e de Campo de Gramíneas. O experimento foi desenvolvido no Pantanal sul-mato-grossense, no Município de Corumbá, MS, no período de outubro a novembro de 2006. Cupins do gênero Nasutitermes, serviram de iscas. Em cada área, 80 indivíduos dessa espécie permaneceram expostos no solo sobre uma base de papel filtro durante 10 Minutos. Observou-se a presença de 15 morfoespécies pertencentes a seis gêneros e cinco subfamílias, sendo mais freqüente Myrmicinae. No campo de gramíneas ocorreram maior riqueza e diversidade de espécies. Wasmania sp.1 foi responsável pelo maior número de iscas removidas na Mata e Dorymyrmex sp. 1 no Campo de Gramíneas. A diferença no tempo médio para remoção das iscas nas duas áreas (mata e gramínea) não foi significativa. Das 160 iscas oferecidas durante o experimento, 115 sofreram atacadas por formigas: 69 na mata e 46 no campo de gramínea.

Palavras-Chave: Atividade de forrageamento, formigas, Pantanal, predação.

\section{Activity of Ants (Hymenoptera: Formicidae) Foraging in a Forest Patch and in a subtropical seasonally flooded lowland grassland in the Pantanal from Mato Grosso do Sul, Brazil}

Abstract. The main goal of this research was to evaluate the exploitation rate of live baits for ants in an environment of forest patches and subtropical seasonally flooded lowland grassland. The samples were collected from October to November 2006 in the Pantanal, sub-regions Miranda and Abobral, municipality of Corumbá, Mato Grosso do Sul, Center West Brazil. Termites of the genera Nasutitermes were used as bait, and remained exposed for 10 minutes on the soil. Eighty baits were used in each environment. Fifteen ant morphospecies in six genera and five subfamilies were collected, and the Myrmicinae were the most frequent subfamily. We found larger richness and diversity of species in the subtropical seasonally flooded lowland grassland. Wasmania sp.1 was responsible for the largest number of removed baits (30) in the Forest Patch and Dorymyrmex sp.1 in the subtropical seasonally flooded lowland grassland. The difference in the average time for removal of the baits from the two areas was not significant. From the 160 baits offered during the experiment, 115 were attacked by ants, 69 in the Forest Patch and 46 in the grassland.

Key words: Ants, foraging activity, Pantanal, predation

stima-se que existam cerca de 15.000 a 20.000 espécies de formigas no planeta (HölLDOBLER \& WiLSON 1990), sendo 3.000 a 8.000 na região neotropical. Elas compõem cerca de quatro vezes a biomassa dos vertebrados em unidades de superfície nas matas sul-americanas (HöLLDobler \& WiLson 1994).

O forrageamento de formigas é uma atividade de seleção, corte e transporte de pequenos pedaços de vegetais para o interior de uma colônia. Diferentes espécies de cortadeiras têm um horário preferencial específico, com movimentos orientados química ou fisicamente (DELLA LúcIA et al. 1993).

Em estudos de diversidade e similaridade da mirmecofauna edáfica em vários ecossistemas do cerrado (floresta de galeria, cerradão, cerrado, cultura de eucalipto, pastagens e culturas de soja) TAVAREs et al. (2001), observaram que a diversidade encontrada nos ecossistemas antrópicos foi inferior àquela observada para os ecossistemas naturais em todas as localidades estudadas. Eles sugeriram que a baixa diversidade comparativa desses ecossistemas pode ser conseqüência das práticas de implantação das culturas e do próprio ambiente simplificado das mesmas.

Trabalhos realizados por VASCONCELOS (1999) demonstraram que os altos níveis de perturbação do habitat resultaram em uma diminuição na riqueza de espécies e aumento na abundância de formigas. Esse resultado deve-se provavelmente ao fato de predominarem em ambientes degradados espécies como Pheidole e Solenopsis, que possuem ampla adaptabilidade a ambientes perturbados além de apresentarem um recrutamento maciço no forrageamento.

Pesquisas realizadas em monocultura de Eucalyptus revelaram uma redução da riqueza e diversidade de formigas não desfolhadoras em comparação com uma reserva de cerrado "stricto sensu". Em decorrência da homogeneidade da vegetação, os locais de nidificação e a disponibilidade de presas diminuíram (TAVAREs et al. 2001).

Resultados obtidos por Moutinho (1998) em áreas de floresta primária, secundária e de pastagens abandonadas revelaram um total de 54 espécies para floresta primária, 56 espécies para floresta secundária e 25 para o pasto abandonado. Ele concluiu que quando florestas são convertidas em pastagens há uma drástica redução (cerca de 50\%) na diversidade das espécies de formigas. Em contrapartida, algumas espécies, principalmente as de hábitos generalistas são favorecidas pela conversão de florestas em pastagens.

Em habitats muito homogêneos, como os de cultivos anuais, terrenos urbanos e campos de gramíneas, o número de espécies é baixo e existe forte dominância de uma delas ou mais. Em florestas e outros habitats com forte estratificação vertical, esse número é elevado e a dominância relativa delas é baixa (Majer \& Delabie 1994).

Uma floresta de rápido crescimento, a de Eucalyptus spp., utilizada no Brasil na produção de carvão vegetal, celulose, papel, chapas e painéis, apresentam o problema das formigas cortadeiras. O plantio homogêneo favorece o desenvolvimento de formigas do gênero Atta e Acromyrmex, consideradas pragas florestais nas Américas (Delda Lúcia et al. 1993).

Oliveira et al. (1995) relataram que há variação na diversidade de formigas influenciada pelas características do ambiente. No entanto, MARINHO (2002), estudando a diversidade de formigas da serrapilheira em eucaliptais e áreas de cerrado do Estado e Minas Gerais, encontrou 67 espécies na área de vegetação nativa e 133 na área de eucaliptais. 
Até recentemente, o desmatamento do planalto central brasileiro adjacente ao Pantanal era considerado a maior ameaça para essa área. Atualmente o desmatamento dentro da planície é um problema mais crítico: cerca de $40 \%$ das florestas e savanas foram removidas para a formação de pastagens, tornando os ambientes simplificados, freqüentemente com a introdução de gramíneas exóticas (HARRIS et al. 2005).

O presente estudo teve por objetivo verificar a atividade de forrageamento e a similaridade das comunidades de formigas em ambientes de mata e campo de gramínea na sub-região do Pantanal de Miranda-Abobral.

\section{Material e Métodos}

O estudo foi desenvolvido no Pantanal do Estado de Mato Grosso do Sul, MS, próximo à base de estudos do Pantanal (BEP) da Universidade Federal de Mato Grosso do Sul, localizada na sub-região do Pantanal do Miranda-Abobral, município de Corumbá - MS $\left(19^{\circ} 35^{\prime} \mathrm{S}, 57^{\circ} 2^{\prime} \mathrm{W}\right)$, no período de outubro a novembro de 2006.

Utilizaram-se duas áreas para a realização do experimento: uma de mata, localizada próximo à margem do rio Miranda, caracterizada por vegetação de grande porte, categoria estrutural arbórea fechada e outra, um Campo de Gramínea, próximo ao rio Miranda, composto por gramíneas rasteiras, dificilmente ultrapassando $15 \mathrm{~cm}$ de altura, utilizado comumente para pastagem por bovinos e de animais silvestres.

Iscas de cupins operários do gênero Nasutitermes foram colocados em cima de uma base de papel filtro com $10 \mathrm{~cm}$ de diâmetro, permanecendo expostos sobre o solo por 10 minutos. As formigas que atacaram as iscas eram coletadas e colocadas em vidros com álcool 70\%, para posterior identificação, segundo chave dicotômica de BoLton (1994).

Quatros Coletas (duas na mata e duas no campo de gramíneas) foram realizadas em transectos localizados a uma distancia mínima de 40 metros da margem do rio e eqüidistantes entre si. Cada transecto continha 10 iscas, perfazendo um total de 80 iscas em cada ambiente.

Para comparação do tempo de remoção das iscas nos ambientes estudados, foram utilizados os testes de t-Student para as variáveis contínuas e para o número de remoções utilizou-se o qui-quadrado $\left(\chi^{2}\right)$, ambos com probabilidade de $5 \%$. Os resultados, as médias, desvio padrão e número amostral são apresentados respectivamente como (x) (sx) (n).

Para determinação da similaridade entre os dois ambientes estudados, ou seja, a semelhança das espécies responsáveis pelo forrageamento frente a iscas oferecidas, utilizou-se o índice de Mountford, proposto para indicar a semelhança entre os dois ambientes, com relação à composição de seus indivíduos, conforme a seguinte fórmula:

$$
\text { IS }=\frac{2 j}{2 a b-(a+b) \times j}
$$

onde: a: número de espécies no habitat a; b: número de espécies no habitat $b$; $j$ : número de espécies comuns aos dois habitats.

\section{Resultados}

Foram encontradas 15 morfoespécies pertencentes a seis gêneros e cinco subfamílias. A subfamília mais freqüente foi Myrmicinaecomnovemorfoespécies. Formicinae e Dolichoderinae aparecem em seguida com duas. Pseudomyrmecinae e Ponerinae foram representadas apenas por uma morfoespécie cada (Tabela 1).

Dos sete gêneros registrados, o mais rico em número de morfoespécies foi Pheidole com sete morfoespécies, seguido de Camponotus e Dorymyrmex com duas, Pseudomyrmex, Ectatomma, Wasmania e Crematogaster foram representados apenas por uma morfoespécie (Tabela 1).

Das 160 iscas oferecidas durante o experimento, 115 foram atacadas por formigas $(71,9 \%)$, sendo que 69 iscas foram removidas na mata $(86,25 \%)$ e 46 no campo de gramínea $(57,5 \%)$. Esta diferença foi altamente significativa $\left(\chi^{2}=17,574\right.$; $\mathrm{p}=0,000)$.

Observou-se maior riqueza de morfoespécies ocupando as iscas no campo de gramínea ( $\mathrm{S}=11)$, enquanto na mata a riqueza foi de seis morfoespécies ( $\mathrm{S}=6$ ). (Tabela 1). Apenas duas espécies foram comuns para os dois ambientes, demonstrando uma baixa similaridade entre eles (Montford $=0,04$ ).

Verificou-se uma maior freqüência de remoção de iscas pela morfoespécie Wasmania sp.1 (30 iscas), seguido de Pheidole sp.3, com 24 para o ambiente de mata. No campo de gramínea Dorymyrmex sp.1 foi responsável pelo maior número de remoção, 23, seguido de Pheidole sp.2 com oito (Tabela 1).

O tempo médio para remoção das iscas foi maior na mata com média $(3,03)$, desvio padrão $(2,40)$ e número amostral de 69 respectivamente, enquanto na gramínea foi de $(2,89)$ $(2,20)(45)$, no entanto a diferença não foi significativa $(t=0,298$, $\mathrm{p}=0,73)$.

Tabela 1. Registros de morfoespécies de formigas encontradas removendo iscas de cupins nas duas áreas estudadas, na região do Pantanal, no período de outubro a novembro de 2006.

\begin{tabular}{|c|c|c|c|}
\hline \multirow[b]{2}{*}{ Subfamílias } & \multirow[b]{2}{*}{ Morfoespécies } & \multicolumn{2}{|c|}{ Ocorrência } \\
\hline & & $\begin{array}{c}\text { Mata } \\
(n=80)\end{array}$ & $\begin{array}{l}\text { Campo } \\
(n=80)\end{array}$ \\
\hline \multirow[t]{9}{*}{ Myrmicinae } & Wasmania sp.1 & 30 & - \\
\hline & Pheidole sp.2 & 6 & 8 \\
\hline & Pheidole sp.3 & 24 & 3 \\
\hline & Pheidole sp.4 & 7 & - \\
\hline & Pheidole sp.5 & - & 2 \\
\hline & Pheidole sp.6 & - & 1 \\
\hline & Pheidole sp.7 & - & 3 \\
\hline & Pheidole sp.8 & - & 1 \\
\hline & Crematogaster sp.1 & - & 2 \\
\hline Pseudomyrmecinae & Pseudomyrmex sp.1 & - & 1 \\
\hline \multirow[t]{2}{*}{ Formicinae } & Camponotus sp.1 & 1 & - \\
\hline & Camponotus sp.2 & - & 1 \\
\hline \multirow[t]{2}{*}{ Dolichoderinae } & Dorymyrmex sp.1 & - & 23 \\
\hline & Dorymyrmex sp. 2 & - & 1 \\
\hline Ponerinae & Ectatomma sp.1 & 1 & - \\
\hline Total & & 69 & 46 \\
\hline
\end{tabular}

\section{Discussão}

AmaiorfreqüênciadeformigasdasubfamíliaMyrmicinae responsáveis pela remoção das iscas deve-se provavelmente ao fato desta subfamília ser numerosa e conter espécies onívoras de hábitos variados. Segundo Fowler et al. (1991), esta subfamília é a mais abundante e apresenta maior diversidade de hábitos na região neotropical e no mundo. Em levantamentos feitos em regiões neotropicais foi encontrada elevada quantidade de espécies de Myrmicinae. Esta é a maior da subfamília Formicidae, com mais de $55 \%$ das espécies encontradas no mundo, seguida por Formicinae e Dolichoderinae (Lopes \& SANTOS 1996). Vários estudos citam esta subfamília como a mais freqüente (CASTRO \& Queiroz 1987; Roth et al. 1994; Moutinho 1998).

A maior ocorrência de morfoespécies de Pheidole, comparado com as demais (Camponotus, Dorymyrmex, Pseudomyrmex, Ectatomma, Wasmania e Crematogaster) provavelmente deve-se ao fato de este gênero apresentar elevado número de espécies. Segundo, MARINHo (2002), estudando a diversidade de formigas do cerrado em sete localidades distintas, encontraram-se espécies do gênero Pheidole em todos os ambientes amostrados. Concluiu que este gênero foi o mais rico em morfoespécies juntamente com Camponotus. Vários estudos 
apontam Pheidole como gênero com o maior número de espécies (Majer \& Delabie 1994; Verhaagh \& Rosciszewski 1994; Soares et al. 1998; MARINHO et al. 2002).

Além disso, espécies pequenas como Pheidole apresentam recrutamento maciço, o que facilita a localização da fonte de alimento, garantindo maior efetividade no forrageamento. Devido à limitação em dominar e carregar recursos, formigas pequenas teoricamente devem recrutar para garantir sua dominação da reserva alimentar, evitando sua perda para uma formiga maior. A velocidade de recrutamento entre espécies menores deve ocorrer com celeridade que em formigas maiores (FUTUYMA 1986).

Muitas espécies de Myrmicinae, em especial do gênero Pheidole, são importantes predadoras de cupins, enquanto espécies de Crematogaster são predadoras ocasionais. O gênero Pheidole apresenta uma ampla adaptação, podendo ser encontrado desde ambientes naturais até ambientes mais perturbados, apresentando um comportamento agressivo com recrutamento eficiente e massivo (HöLLDOBLER \& WILSON 1990).

De acordo com o índice de Mountford, a baixa similaridade, somada à diferença de morfoespécies encontradas dominando as iscas nos dois ambientes estudados, pode ser explicada pela diferença da complexidade vegetal dos ambientes, o que contribui para a existência de nichos diferenciados em cada ambiente. Portanto, formigas que são dominantes em ambientes heterogêneos, como mata, podem não o ser em ambientes homogêneos, devido à competição e diferenciação dos nichos.

A maior riqueza de espécies observada no campo de gramínea ocorre provavelmente em virtude dste ambiente possuir uma menor oferta de alimento, estimulando o forrageamento por um número maior de espécies e aumentando a competição pelas iscas oferecidas. Tal resultado corrobora os dados obtidos por MARINHO et al. (2002). Contradizendo a maior parte dos trabalhos sobre o assunto, que encontraram maior riqueza de espécies em eucaliptais do que em áreas de cerrado, inferindo que a riqueza de formigas numa região não depende somente dos ambientes estudados. BARbosa \& Fernandes (2003) também encontraram resultados semelhantes quando compararam o forrageamento de formigas em áreas de Eucaliptus urophylla, Blake, com e sem manejo. Observaram que na área com manejo e, portanto sem sub-bosque, ocorreu maior porcentagem de iscas retiradas por formigas.

A baixa similaridade e diferença na riqueza somada à diferença significativa no número de iscas removidas nos ambientes estudados, reforçam a hipótese de que a complexidade do habitat pode influenciar na abundância e riqueza de formigas responsáveis pela remoção das iscas.

Em ambientes heterogêneos, tendo em vista uma maior oferta de alimento e forte estratificação vertical, a localização da fonte alimentar pode ser dificultada, favorecendo a presença de nichos diversificados e diminuindo a competição por recursos. Desta forma, apenas algumas espécies se destacam na localização da presa. De acordo com BARBOSA \& FERNANDES (2003) em ambientes que apresentam maior complexidade vegetal proporcionam um maior nicho de nidificação e itens alimentares para as formigas.

Explica-se a maior riqueza do ambiente de gramínea em função da utilização de iscas vivas de cupim. Este método não é mais adequado para se obter dados de riqueza, porque proporciona, principalmente, a observação de competição de diferentes espécies de formigas sobre as iscas. Estudos realizados por Santana-Reis \& Santos (2001), utilizando isca de atum, em quatro ambientes distintos, uma área degradada, uma pastagem de Brachiaria decubens Stapf um campo de Turnera ulmifolia Linn. e uma área de bosque de Caesalpinia echinata Lam, concluíram que o aumento do número de espécies presentes nas comunidades é devido ao aumento da complexidade da estrutura dos habitats. Neste estudo a área degradada foi a que apresentou a menor riqueza de espécies e a área do bosque a maior.

BARBOSA \& FERNANDES (2003) observaram maior riqueza, diversidade e equitabilidade para a fauna de formigas em áreas de E. urophylla mais complexas, com presença de sub-bosque; entretanto, em experimento com oferecimento de iscas, observaram que a maior proporção de retirada ocorreu nas áreas com manejo e sem a presença de sub-bosque. Isso foi explicado porque a área com maior complexidade vegetal disponibilizava maior quantidade de recursos alimentares, evitando conseqüentemente, competição pelas iscas oferecidas.

Apesar de não ter sido observado diferença significativa, o tempo para remoção da isca foi maior na mata, o que provavelmente ocorre pela dificuldade de localização da presa nesse ambiente. Devido à complexidade vegetal e forte estratificação, existência de maior oferta de alimento. CoRRÊA et al. (2006), em estudos no pantanal da Nhecolândia, observaram uma relação positiva entre a riqueza da comunidade de formigas e a densidade da vegetação herbácea, corroborando a idéia de que a heterogeneidade do ambiente é um fator determinante para a coexistência e diminuição de competição entre espécies.

As formigas, durante 100 milhões de anos de coexistência com os cupins, têm-se destacado como suas principais inimigas naturais. No entanto, a atividade de forrageamento das formigas modifica-se em razão de mudanças ambientais da demanda da colônia (HÖLLDOBLER \& WILSON 1990).

O presente estudo demonstrou que uma diferença quanto à riqueza da fauna e à atividade de forrageamento das formigas é, provavelmente, decorrente da complexidade vegetal e das condições bióticas e abióticas a que é submetido cada um dos ambientes como, por exemplo, alagamento sazonal e presença constante de gado na área de gramíneas.

\section{REFERÊNCIAS}

Barbosa, L.P. \& W.D. Fernandes, 2003. Bait removal ants (Hymenoptera: Formicidae) in managed and unmanaged Eucalyptus urophylla S.T. BLAKE fields. Brazilian Journal Ecology, 5 e 6: 61-63.

Bolton, B., 1994, Identification guide to the ant genera of the world. Harvard University Press, Cambridge, MA.

Castro, A.G. \& M.V.B. Queiroz, 1987. Estrutura e organização de uma comunidade de uma comunidade de formigas em agroecossistema neotropical. Anais da Sociedade Entomológica do Brasil, 16: 363-375.

Correa, M.M., W.D. Fernandes \& I.R. Leal, 2006. Diversidade de formigas epigéicas (Hymenoptera: Formicidae) em capões do Pantanal Sul Matogrossense: relações entre riqueza de espécies e complexidade estrutural da área. Neotropical Entomology, 35: 724-730.

Della Lúcia, T.M.C. \& M.A. Oliveira, 1993. As Formigas Cortadeiras. Viçosa: Folha de Viçosa, 262 p.

Fowler, H.G., L.C. Forti, C.R.F. Brandão, J.H.C. Delabie \& H.L. Vasconcelos, 1991. Ecologia nutricional de formigas, p. 131-223. In: Panizzi, A.R. \& J. R. P. Parra (Eds.). Ecologia nutricional de insetos e suas implicações no manejo de pragas. São Paulo, Manole, 359p.

Futuyma, D.J., 1986. Evolutionary Biology. Sinauer Associates, Sinderland, Massachusetts, 6oop.

Harris, M.B., W.M. Tomas, G. Mourão, C.J. da Silva, E. Guimarães, F. Sonoda, \& E. Fachim, 2005. Desafios para proteger o Pantanal brasileiro: ameaças e iniciativas em conservação. Megadiversidade, 1: 156-163.

Hölldobler, B. \& E.O. Wilson, 1990. The ants. Harvad University Press, 732p.

Hölldobler, B. \& E.O. Wilson, 1994. Journey of the Ants: A Story of Scientific Exploration. Cambridge, Massachusetts: Harvard University Press, 228p.

Lopes, B.C. \& R.A, Santos, 1996. Aspects of the ecology of ants (Hymenoptera: Formicidae) on the mangrove vegetation of Rantones. Santa Catarina Island, SC, Brazil. Boletim Entomológico Venezuelano, 11: 123.

Marinho, C.G.S., R. Zanetti \& J.H.C. Delabie, 2002. Ant (Hymenoptera: Formicidae) diversity in Eucalyptus (Myrtaceae) plantations and cerrado litter in Minas Gerais, 
Brazil. Neotropical Entomology, 31: 187-195.

Majer, J.D. \& J.H.C. Delabie, 1994. Comparision of the of the ant communities of annually inundated and terra firme forests at Trombetas in Brazilian Amazon. Insects Sociaux 41: 343-359. Moutinho, P.R.S. 1988. Impactos da formação de pastagens sobre a fauna de formigas:conseqüências para a recuperação florestal na Amazônia Oriental. p.155-170. In: Gascon, C.; Moutinho, P. (eds). Floresta Amazônica: dinâmica, regeneração e manejo. Manaus-AM, INPA.

Oliveira, M.A., T.M.C. Della Lucia, M.S. Araújo \& A.P. da Cruz, 1995. A fauna de formigas em povoamentos de eucalipto na mata nativa no estado do Amapá. Acta Amazonica, 25: 117126.

Roth, D.S.; I. Perfecto \& B. Rathcke, 1994. The effects of management systems on ground-foraging ant diversty in Costa-Rica. Bulletin Ecological Sociology of America, 4: 423436.

Santana-Reis, V.P.G. \& G.M.M. de Santos, 2001. Influência da estrutura do habitat em comunidades de formigas em Feira de Santana, Bahia, Brasil. Sitientibus, 1: 66-70.

\section{Como citar este artigo:}

Sant'Ana, M.V., R.B.R. Trindade, C.C.S. Lopes, O. Faccenda \& W.D.

Fernandes. 2008. Forrageamento de Formigas (Hymenoptera:

Formicidae) em Áreas de Mata e Campo de Gramíneas no

Pantanal sul-mato-grossense. EntomoBrasilis, 1(2): 29-32.

www.periodico.ebras.bio.br/ojs
Soares, S.M., C.G.S. Marinho \& T.M.C. Della Lucia, 1998. Diversidade de invertebrados edáficos em áreas de eucalipto e mata secundária. Acta Biologica Leopoldensia, 19: 157-164.

Tavares, A.A.; P.C. Bispo \& A.C.S. Zanzini. 2001. Comunidades de formigas epigéicas (Hymenoptera, Formicidae) em áreas de Eucalyptus cloeziana F. Muell e de vegetação nativa numa região de cerrado. Revista Brasileira de Entomologia, 45: 251256.

Vasconcelos, H.L., 1999. Effects of forest disturbance on the structure of ground-foraging ant communities in central Amazonia. Biodiversity and Conservation: 407-418.

Verhaagh, M. \& K. Rosciszewski, 1994. Ants (Hymenoptera: Formicidae) and savanna in the Biosphere Reserve Beni, Bolivia. Andrias, 13: 199-214.

Recebido em: 04/03/2008

Aceito em: 01/07/2008

$* * * * * * * * * * * * *$
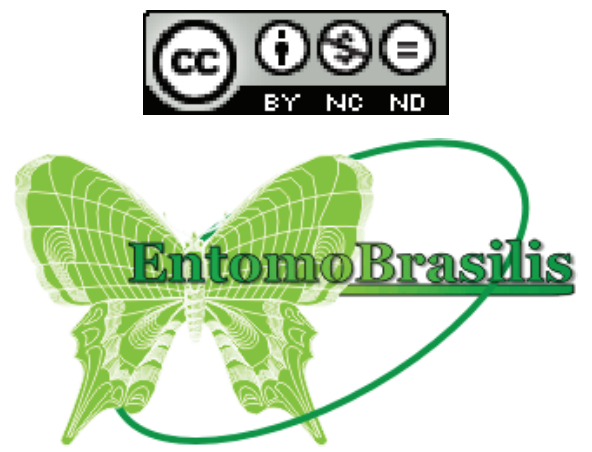